\title{
Padrão de oclusão em prótese Protocolo de Brånemark: uma revisão de literatura
}

\author{
Standard of occlusion in Brånemark Protocol prothesis: \\ a literature review
}

\author{
Caio César Vieira Rocha* \\ Manassés Tercio Vieira Grangeiro* \\ Viviane Maria Gonçalves de Figueiredo ${ }^{* * *}$
}

\section{Resumo}

O padrão oclusal pode ser considerado um fator crítico para a longevidade dos componentes do sistema estomatognático como também das reabilitações implantossuportadas. Objetivo: revisar a literatura em relação ao padrão de oclusão mais adequado para reabilitar um paciente edêntulo com prótese Protocolo de Brånemark. Revisão de literatura: nesta revisão, os artigos de estudos clínicos retrospectivos (2 artigos) e in silico (2 artigos) foram incluídos. Considerações finais: padrões de oclusão em prótese Protocolo de Brånemark são um tema muito escasso na literatura; o uso de uma oclusão mutuamente protegida com guia lateral em canino favorece a distribuição da tensão sobre os implantes e o osso alveolar; no entanto, a condição do arco antagonista pode influenciar esta escolha.

Palavras-chave: Implante dental. Oclusão. Protocolo de Brånemark.

\section{Introdução}

Os implantes osseointegrados apresentam grande avanço na Odontologia; as próteses implantossuportadas têm ganhado popularidade ao longo dos anos em relação ao uso de próteses totais convencionais, em decorrência de a reabilitação implantossuportada favorecer a estabilidade e a retenção. ${ }^{1}$

Apesar da confiabilidade e das altas taxas de sucesso relacionadas aos tratamentos com implantes, o afrouxamento dos parafusos de retenção dos pilares apresenta-se como uma falha frequente. ${ }^{2} \mathrm{~A}$ natureza do afrouxamento é complexa, e os mecanismos responsáveis por tais falhas ainda não estão totalmente elucidados. Também a fratura da extensão do cantiléver está entre as principais causas do insucesso da prótese tipo Protocolo de Brånemark. Quando o cantiléver apresenta grande extensão, aumenta o risco da fratura da prótese, do implante ou do pilar mais distal. ${ }^{3}$

O padrão oclusal pode ser considerado um fator crítico para a longevidade dos componentes do sistema estomatognático como também das reabilitações implantossuportadas. Na dentição natural, o ligamento periodontal atua como um sistema de amortecimento, que melhora a absorção de tensões oclusais. No entanto, na implantodontia, não há uma estrutura semelhante ao ligamento periodontal na interface implante-osso, ou seja, para absorção da carga oclusal. Assim, o estresse oclusal é totalmente transmitido pelas estruturas rígidas da prótese e do implante ao osso residual. Caso as forças oclusais excedam a capacidade do sistema, a reabilitação oral falha, devido à sobrecarga. ${ }^{4}$ 
Com base no exposto, objetivou-se revisar a literatura acerca do padrão de oclusão mais adequado para reabilitar pacientes edêntulos com prótese Protocolo de Brånemark.

\section{Materiais e método}

As bases de dados utilizadas nesta revisão são detalhadas no Quadro 1. Foi realizada uma busca de palavras-chave no Medical Subject Headings, e foram selecionadas as seguintes: implante dental; oclusão; e Protocolo de Brånemark. Os critérios de inclusão e exclusão dos artigos científicos são apresentados na Quadro 2. A partir da leitura de resumos ou abstracts, a seleção do conteúdo foi conduzida com base nos critérios estabelecidos. A Figura 1 exibe o número de artigos presente em cada base de dados consultada e o número de artigos incluídos e excluídos da revisão.

Quadro 1 - Bases de dados e respectivos sites

\begin{tabular}{|c|c|}
\hline Base de dados & Site \\
\hline PubMed & $<$ http://www.ncbi.nlm.nih.gov/pubmed $>$ \\
\hline SciELO & $<$ www.scielo.org $>$ \\
\hline Bireme & $<$ bvsalud.org $>$ \\
\hline Lilacs & $<$ http://lilacs.bvsalud.org/> \\
\hline Cochrane Library & $<$ http://www.cochranelibrary.com/> \\
\hline
\end{tabular}

Fonte: autores.

Quadro 2 - Critérios de inclusão e exclusão utilizados na revisão de literatura

\begin{tabular}{|c|c|}
\hline Critérios de inclusão & Critérios de exclusão \\
\hline $\begin{array}{c}\text { Estudos in vitro e in silico, ensaios } \\
\text { clínicos, revisões sistemáticas, } \\
\text { metanálise. }\end{array}$ & $\begin{array}{c}\text { Caso clínico, carta ao editor, } \\
\text { artigo de opinião (baixa } \\
\text { evidência científica) }\end{array}$ \\
\cline { 1 - 1 } \begin{tabular}{c} 
Artigos sobre a temática em estudo \\
\cline { 1 - 1 } $\begin{array}{c}\text { Artigos escritos em português, } \\
\text { inglês e espanhol }\end{array}$
\end{tabular} & $\begin{array}{c}\text { Artigos sobre implantes } \\
\text { unitários }\end{array}$ \\
\hline
\end{tabular}

Fonte: autores.

Figura 1 - Fluxograma do estudo realizado

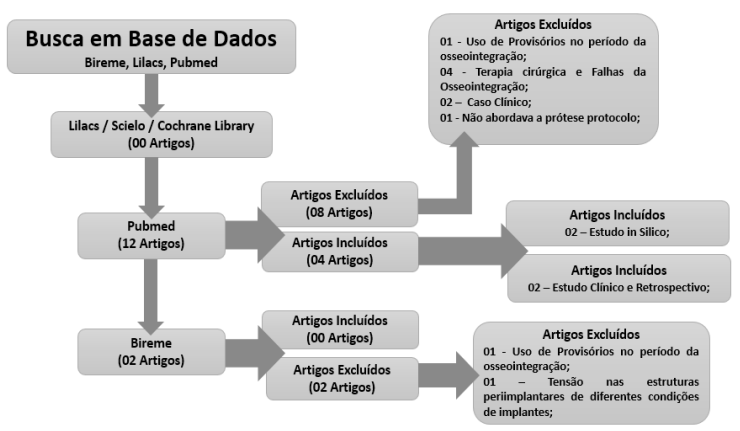

Fonte: autores.

\section{Revisão da literatura}

\section{Prótese Protocolo de Brånemark}

Inicialmente a prótese Protocolo de Brånemark foi preconizada com a inserção de cinco a seis implantes na maxila e na mandíbula totalmente edêntulas, para fixação da prótese implantossuportada. Geralmente as regiões posteriores apresentam alguma indisponibilidade óssea, devido a isso, uma estabilidade anterior adequada deve ser alcançada, para suportar as extensões dos cantilévers bilaterais que se estendem distalmente. ${ }^{5}$

As complicações mais prevalentes em prótese sobre implante são falhas na osseointegração do implante, fratura do pilar e do parafuso protético, fratura do cantiléver, afrouxamento do parafuso protético. $^{6}$

\section{Oclusão em implantodontia}

O controle biomecânico da oclusão está relacionado com o sucesso clínico das reabilitações com próteses sobre implante. Os implantes respondem às cargas oclusais de maneira distinta do elemento dental, devido à ausência do ligamento periodontal. Sabe-se que as cargas oclusais são consideradas uma das principais causas das complicações biomecânicas relacionadas às falhas no tratamento implantar após a colocação dos implantes em oclusão. Com isso, é necessário que o cirurgião-dentista conheça os meios e os fatores que fazem com o que as cargas oclusais afetem os implantes e como tais tensões podem influenciar na sobrecarga recebida nas próteses sobre implantes, com o intuito de selecionar um esquema oclusal ideal para cada caso individualmente. $^{7}$

A importância de padrões oclusais como coadjuvantes na estabilidade e na retenção da prótese total convencional tem sido discutida; o arranjo oclusal é um fator crítico para o sucesso dessa reabilitação mucossuportada, pois interfere na reabsorção óssea do rebordo residual, no conforto durante a mastigação e na segurança ao utilizar as próteses, também favorece a qualidade de vida do paciente. ${ }^{8}$

O uso da desoclusão por guia canino é considerado o tipo de desoclusão ideal na dentição natural, em que, nos movimentos de lateralidade direita e esquerda, deve ocorrer apenas o contato dos caninos em lado de trabalho; enquanto o lado de não trabalho ou balanceio não deve apresentar contato dental (Figura 2). Já, no movimento excursivo de protusão, os dentes anteriores, quando se tocam, desocluem os posteriores, assim a busca pela oclusão mutuamente protegida é um dos maiores motivos para optar-se por esse tipo de padrão oclusal. ${ }^{9}$ 
Figura 2 - Esquema do movimento de lateralidade de uma prótese Protocolo de Brånemark com padrão de oclusão mutuamente protegida (guia pelo canino)

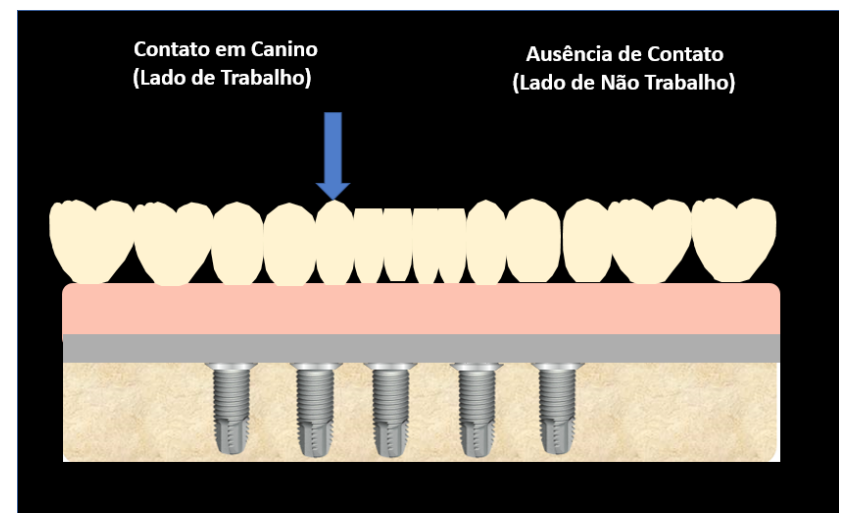

Fonte: autores.

$\mathrm{Na}$ oclusão balanceada bilateral, deve haver contatos bilaterais harmônicos entre os arcos antagonistas tanto no lado de trabalho quanto no de balanceio ou não trabalho, nas posições cêntricas e excêntricas (Figura 3). ${ }^{1} \mathrm{~A}$ ideia principal, em relação ao padrão de oclusão balanceada bilateral, se dá pela estabilidade das próteses por meio dos contatos bilaterais durante a função, ou seja, por movimentos excursivos da mandíbula. ${ }^{9}$

Figura 3 - Esquema do movimento de lateralidade de uma prótese Protocolo de Brånemark com padrão de oclusão balanceada bilateral

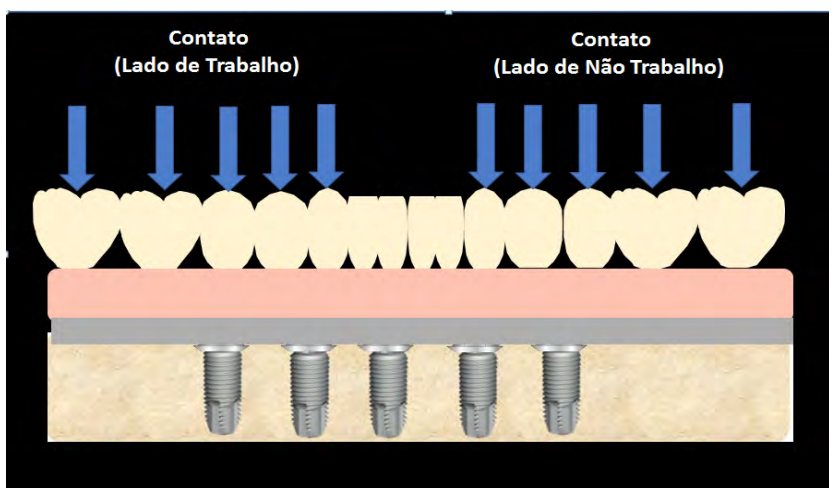

Fonte: autores.

A seleção do padrão de oclusão ou da filosofia oclusal a ser utilizada pelo cirurgião-dentista, ao reabilitar um paciente com uma prótese Protocolo de Brånemark, depende principalmente da estratégia reabilitadora do arco antagonista, a fim de favorecer a estabilidade e a retenção com auxílio do arranjo oclusal ${ }^{1}$ (Quadro 3).
Quadro 3 - Padrão oclusal versus arco antagonista para prótese Protocolo de Brånemark ${ }^{3}$

\begin{tabular}{|c|c|c|}
\hline $\begin{array}{c}\text { Reabilitação } \\
\text { implantossuportada }\end{array}$ & Arco antagonista & Padrão oclusal \\
\hline \multirow{4}{*}{ 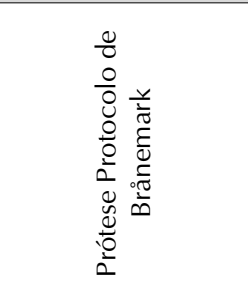 } & Dentição natural & $\begin{array}{c}\text { Oclusão mutuamente } \\
\text { protegida }\end{array}$ \\
\hline & $\begin{array}{c}\text { Prótese total } \\
\text { mucossuportada }\end{array}$ & $\begin{array}{c}\text { Oclusão balanceada } \\
\text { bilateral }\end{array}$ \\
\hline & $\begin{array}{c}\text { Prótese Protocolo de } \\
\text { Brånemark }\end{array}$ & $\begin{array}{c}\text { Oclusão mutuamente } \\
\text { protegida }\end{array}$ \\
\hline & Overdenture & $\begin{array}{c}\text { Oclusão balanceada } \\
\text { bilateral }\end{array}$ \\
\hline
\end{tabular}

Fonte: autores.

\section{Discussão}

A dúvida quanto à seleção do padrão de desoclusão utilizado em uma prótese Protocolo de Brånemark está associada à condição de que tal reabilitação protética é uma prótese total, porém suportada por implantes, assim a transmissão de força é do tipo implantossuportada. As próteses implantossuportadas totais são, na verdade, a união dos conceitos de prótese total com suporte tecidual e próteses fixas dentossuportadas. ${ }^{2}$ Então, que filosofias oclusais seguir? Uma oclusão mutuamente protegida, pertencente à oclusão ideal? Contudo, e se o paciente a ser reabilitado for edêntulo? Uma oclusão balanceada bilateral, desde que a prótese que esse paciente receba seja uma prótese total?

Esse dilema oclusal está ganhando mais espaço na comunidade científica nos últimos anos, pois muito estudou-se sobre estratégias cirúrgicas, a fim de estabelecer a osseointegração; agora, após a consagração deste processo, vem se buscando entender os motivos das complicações e falhas de reabilitações sobre implantes, sendo os fatores oclusais um dos menos pesquisados, com base nas buscas realizadas nas bases de dados adotadas nesta revisão.

Conforme os dados levantados, a base Bireme apresentou apenas duas literaturas, e uma foi excluída, pois não estava de acordo com o tema; nas bases Lilacs, SciELO e Cochrane Library, não foram encontradas literaturas sobre o tema. Por fim, na base PubMed, 12 artigos resultaram da busca, mas apenas quatro abordavam o tema em estudo, o que mostra a pobre evidência sobre o assunto.

Ainda assim, os quatro estudos selecionados são estudos clínicos e retrospectivos, ou seja, acompanhamento de um grupo de pacientes em que se adotou a prótese Protocolo de Brånemark, porém o padrão de oclusão não foi uma variável. Já os demais são estudos in silico, que utilizaram a ferramenta computacional de elementos finitos para compreender as tensões sobre os distintos padrões de oclusão. Estudos laboratoriais apresentaram baixa evidência científica, o que mostra a necessidade de ensaios clínicos randomizados, para consagrar que padrão ou filosofia oclusal deve ser utilizado. 
Observou-se que o padrão de desoclusão mais prevalente, em uma cidade da Noruega, entre pacientes que apresentavam prótese Protocolo de Brånemark, foi a função em grupo $(53,4 \%)$, o guia pelo canino (37\%) e, por fim, a oclusão balanceada bilateral $(9 \%) .^{10}$

Durante estudo retrospectivo de um ano, verificou-se que pacientes com prótese Protocolo de Brånemark e presença de hábito parafuncional (41\%) apresentavam perdidas dos implantes com mais frequência do que pacientes sem parafunção (12\%); nesse sentido, cargas oclusais altas devem ser consideradas um fator de risco. ${ }^{11}$

Em estudo in silico com prótese Protocolo Mandibular, a oclusão mutuamente protegida, com guia lateral pelo canino, apresentou valores de estresse inferiores à oclusão balanceada bilateral. $\mathrm{O}$ uso de próteses Protocolo de Brånemark com extensões de cantilévers distais à oclusão mutuamente protegida deve ser o padrão de escolha para essa reabilitação, por favorecer a distribuição de tensões sobre os implantes e a própria estrutura da prótese, a fim de promover a longevidade da reabilitação. Em contrapartida, a oclusão mutuamente protegida gera três vezes mais tensões por toda a estrutura da prótese. ${ }^{2}$

Modelos modificados do protocolo foram propostos por Brånemark para a reabilitação total sem a presença do cantiléver. Por meio de um estudo in silico, foram feitas as simulações: foram colocados cinco implantes na região entre os forames mentonianos, e dois implantes curtos, em ambos os lados, no setor posterior (em região central de primeiro molar inferior), eliminando, assim, as extensões de cantilévers. Uma força correspondente a $15 \mathrm{~N}$ foi aplicada com um ângulo de $45 \square$ em um dente, representando a oclusão mutuamente protegida; e a mesma força foi aplicada e distribuída em três dentes, para simular a oclusão balanceada bilateral. Foi comparada a magnitude do esforço para cada simulação isoladamente, e observou-se que a oclusão mutuamente protegida, que possui a sua desoclusão pelo canino, gerou tensões mais elevadas no lado de trabalho, em específico, na região do implante curto posterior. Já, na oclusão balanceada bilateral, os resultados foram de forças de intensidade mais baixas e distribuídas mais uniformemente pela estrutura da prótese. Das condições expostas no estudo para o tipo da prótese, a disposição e o número de implantes, o padrão mais apropriado foi o da oclusão balanceada bilateral para a prótese Protocolo de Brånemark com implantes distais, eliminando as extremidades livres. ${ }^{4}$

Talvez a escassez da literatura quanto ao tipo de padrão oclusão mais adequado para prótese Protocolo de Brånemark se dá devido ao sucesso dessa reabilitação implantossuportada. A taxa de sobrevivência global do implante é de 99,5\%. Em estudo clínico retrospectivo de 2 anos, um de 129 pacientes apresentou falhas quanto à osseointegração. Já, em estudo clínico e retrospectivo de 5 anos, observou-se falha apenas de dois implantes em reabilitações com Protocolo de Brånemark, sendo a taxa de sucesso de $95 \% .^{12}$

Outro fator seria a estabilidade satisfatória promovida pela prótese Protocolo de Brånemark, diferentemente do que ocorre com a prótese total mucossuportada, que apresenta falhas em estabilidade e retenção; nesse sentido, há diversos artigos relatados na literatura que buscam, por meio de arranjos oclusais, favorecer o uso desta reabilitação protética. ${ }^{8}$ Por esse motivo, em décadas passadas, utilizavam-se câmaras de succção, a fim de favorecer a retenção e estabilidades de próteses totais superiores.

Com o avanço dos anos e a consagração do uso da prótese Protocolo de Brånemark, será preciso um olhar mais atendo aos padrões de oclusão a serem estabelecidos ao paciente reabilitado. Novos estudos com grau de evidência científica devem ser realizados, a fim de se observar variáveis ainda não analisadas, como a condição do arco antagonista, e estabelecer cientificamente a melhor filosofia oclusal a ser adotada para tais casos clínicos.

\section{Conclusão}

Esta revisão mostra que o padrão de oclusão em prótese Protocolo de Brånemark é um tema muito escasso na literatura. O uso de uma oclusão mutuamente protegida, com guia lateral em canino, favorece a distribuição da tensão sobre os implantes e o osso alveolar. Porém, a condição do arco antagonista pode influenciar a escolha do arranjo oclusal. Por fim, são necessários ensaios clínicos randomizados, que evidenciem a melhor filosofia oclusal, a fim de se obter longevidade na reabilitação oral.

\section{Abstract}

The occlusal pattern can be considered a critical factor for the longevity of the components of the stomatognathic system, as well as of the implantsupported rehabilitations. Objective: to review the literature regarding the most appropriate standard of occlusion to rehabilitate a patient edentulous with Brånemark Protocol prothesis. Literature review: in this review, articles from retrospective clinical studies (02 articles) and in silico studies (02 articles) were included. Final considerations: this literature review shows that standard of occlusion in the Brånemark Protocol prosthesis ns are a very scarce subject in the literature, the use of a mutually protected lateral guiding in canine favors the stress distribution on the implants and alveolar bone, however the condition of the arc antagonist may influence this choice.

Keywords: Dental implant. Occlusion. Brånemark Protocol. 


\section{Referências}

1. Pita MS, Anchieta RB, Ribeiro AB, Pita DS, Zuim PRJ, Pelizzer EP. Fundamentos de oclusão em implantodontia: orientações clínicas e seus determinantes protéticos e biomecânico. Rev Odontol Araçatuba 2008; 29(1):53-9.

2. Greco GD, Jansen WC, Lorenzo JL, Seraidarian PI. Biomechanical analysis of the stresses generated by different disocclusion patterns in an implant-supported mandibular complete denture. J Appl Oral Sci 2008; 17(5):515-2.

3. Pita MS. Estudo biomecânico de conexões implante/pilar com parafusos convencionais e parafusos modificados tipo Cone Morse: análise tridimentional pelo método dos elementos finitos [Tese de Doutorado]. Ribeirão Preto: Universidade de São Paulo; 2013.

4. Greco GD, Las casas EB, Cornacchia TPM, Magalhães CS, Moreira NA. Standard of disocclusion in complete dentures supported by implants without free distal ends: analysis by the finite elements method. J Appl Oral Sci 2012; 20(1):64-9.

5. Naconecy MM. Força e momento fletor em pilares de prótese tipo Protocolo Bränemark em função da inclinação dos implantes distais e do número de pilares [Tese de Doutorado]. Porto Alegre: Faculdade de Odontologia, Pontifícia Universidade Católica do Rio Grande do Sul; 2006.

6. Pelisser A. Falhas mecânicas e biológicas das próteses sobre implantes [Monografia de Especialização]. Florianópolis: Universidade Federal de Santa Catarina; 2003.

7. Sanitá PV, Pinelli LAP, Silva RHBT, Segalla JCM. Aplicação clínica dos conceitos oclusais na implantodontia. Rev Fed Odon 2009; 14(3):268-75.

8. Frota PL, Bastos PL, Figueiredo VMG. Padrão de oclusão em prótese total-um dilema da reabilitação oral. Rev Bah Odont 2016; 7(16):58-62.

9. Cançado CFL. Oclusão balanceada bilateral ou guia canina na reabilitação com prótese total - revisão de literatura. [Monografia de Espeicalização]. Belo Horizonte: Instituto de Estudos da Saúde Sérgio Feitosa; 2008.

10. Wie H. Registration of localization, occlusion and occluding materials for failing screw joints in the Brånemark implant system. Clin Implant Dent Relat Res 1995; 6(1):47-53.

11. Glauser R, Rée A, Lundgren A, Gottlow J, Hammerle CH, Scharer P. Immediate occlusal loading of Brånemarkimplants applied in various jawbone regions: a prospective, 1-year clinical study. Clin Implant Dent Relat Res 2001; 3(4):204-13.

12. Calandriello R, Tomatis M. Immediate occlusal loading of single lower molars using Brånemark System Wide Platform TiUnite ${ }^{\mathrm{TM}}$ implants: a 5-year follow-up report of a prospective clinical multicenter study. Clin Implant Dent Relat Res 2011; 13(4):311-8.

Endereço para correspondência:

Manassés Tercio Vieira Grangeiro

Av. Eng. Francisco José Longo, 777, São Dimas

12245-000, São José dos Campos, SP, Brasil

Telefone: (88) 99906-0612

E-mail: manasses.grangeiro@unesp.br 